\title{
RESEÑA DEL LIBRO: KRASTEV, I. Y HOLMES, S. (2019). LA LUZ QUE SE APAGA. CÓMO OCCIDENTE GANÓ LA GUERRA FRÍA PERO PERDIÓ LA PAZ. BARCELONA: LIBROS DEBATE. 342 PÁGINAS
}

\section{José Antonio Abreu Colombri ${ }^{1}$}

ORCID: 0000-0002-8698-6493

Todos los temas relacionados con la confrontación bipolar estaban fuertemente ideologizados, en todos los campos de especialización de las ciencias sociales. En el caso del pensamiento occidental, la lucha por la propagación de los valores democráticos y los modelos de libertad económica llegó a estar un tanto mitificada en la fase final de la Guerra Fría.

Los ciudadanos del "mundo libre" estaban sometidos a campañas de propaganda tan intensas como en el "mundo comunista", pero en contextos muy diferentes. El alto grado de ideologización, a principios de la década de 1990, consiguió que los discursos políticos fuesen selectivos y, en cierta medida, que los libros de historia contemporánea tuviesen relatos idealizados.

En buena medida, La luz que se apaga ${ }^{2}$ se centra en las cuestiones clave del periodo posterior a la disolución de la Unión Soviética, que marcaron el cambio de tendencia en las mentalidades colectivas. El neoliberalismo, después de convertirse en la corriente de pensamiento dominante en el momento de la victoria final de los sistemas capitalistas, no pudo ejercer un monopolio ideológico consolidado en el mundo de la pos Guerra Fría.

1 Universidad de Alcalá, España. Doctorado en Estudios Norteamericanos. Correo eletrónico: abreucolombri@gmail.com

2 The Light that Failed: A Reckoning (2019, Penguin Books) es el título original del libro. Libros Debate tradujo la publicación de Krastev y Holmes al castellano; es una ramificación empresarial de Penguin Randon House Grupo Editorial (2013). El objetivo de sus diferentes colecciones temáticas es comercializar publicaciones exitosas en el mundo anglosajón en los países hispanohablantes. 
La opinión pública occidental se mostró muy dividida ante el proceso de financiarización y la nueva ortodoxia del libre mercado; los países del antiguo Pacto de Varsovia se sentían sometidos por las imposiciones económicas externas; los otrora estados del tercer mundo consideraban el nuevo orden económico como un humillante estatus neocolonial.

Así, Ivan Krastev ${ }^{3}$ y Stephen Holmes ${ }^{4}$ reflexionan sobre las consecuencias ideológicas del neoliberalismo, aunque el término utilizado sea "modelo liberal". También tratan de establecer un marco analítico sobre el estado de euforia absoluta que se vivió a finales de la Guerra Fría, que tuvo como resultado más visible la aparición de tesis como "el fin de la historia", "la era de la imitación" y "el gran consenso económico". La investigación de estos autores se centra en el segmento temporal de las últimas tres décadas, con constantes menciones referenciales de los acontecimientos previos a la caída del Muro de Berlín.

El libro se articula a través de un apartado introductorio, tres capítulos y una conclusión. La investigación trata de argumentar que los movimientos populistas y los discursos demagógicos, en la actualidad, son consecuencia directa de la actuación política de los actores que instrumentalizan la imagen institucional del sistema democrático. También son planteados como consecuencia indirecta de las imposiciones forzosas del modelo neoliberal en los escenarios postsoviéticos.

A lo largo de todos los apartados de la publicación, trasciende la idea de que la disolución de la Unión Soviética fue el momento culmen de las democracias liberales, pero que, al mismo tiempo, supuso el inicio de un largo periodo de languidecimiento del pensamiento liberal. Es muy interesante ver cómo ambos autores van intercalando casos de estudio concretos sobre contextos históricos previos y análisis de la situación política actual.

3 Es doctor por la Universidad de Sofía. Casi toda su trayectoria profesional ha estado vinculada al mundo del análisis político en el marco de las instituciones de la Unión Europea y de la República de Bulgaria. Actualmente, trabaja en el Instituto de Ciencias Humanas de Viena, el Centro de Estrategias Liberales de Sofía y el Consejo Europeo de Relaciones Exteriores. Tiene una larga lista de publicaciones sobre teoría democrática, relaciones internacionales e historia del proceso de unificación política de la Unión Europea.

4 Es doctor por la Universidad de Yale. Ha impartido clases en las universidades de Harvard, Princeton y Chicago. Al día de hoy, imparte clases en la Facultad de Derecho Walter E. Meyer de la Universidad de Nueva York. También colabora con la Fundación Soros y participa en programas de asesoría política (tanto en los Estados Unidos como en las instituciones de la Unión Europea). Su trayectoria bibliográfica es muy diversa, pero se repiten algunos temas, como los fundamentos jurídicos y filosóficos del pensamiento político contemporáneo y las causas del agotamiento del modelo liberal en el siglo XXI. 
La síntesis teórica de los acontecimientos económicos se convierte en el nexo estructural de toda la investigación, ya que la ejemplificación de los desarrollos conceptuales aparece de forma constante y reiterada. La imitación de los modelos exitosos de Occidente representa el arquetipo descriptivo e interpretativo más utilizado a lo largo de la más de trescientas páginas del libro.

El año 1989 era percibido como uno de los mayores acontecimientos históricos, solo comparable con el inicio del Renacimiento o el colapso del Antiguo Régimen. Occidente se expandía a todas las regiones del planeta, a través de sus corporaciones, las instituciones internacionales y los movimientos culturales. Durante la década de 1990, el fenómeno de la globalización estuvo al servicio de las élites neoliberales, pero el optimismo no duró mucho por las resistencias sociales y por las restricciones resultantes de los atentados del 11-S. La Federación Rusa tardó más de una década en pasar de superpotencia global en descomposición a constituirse en un poder regional pujante.

El colapso financiero de 2008 desencadenó, aunque no de forma inmediata, la mayor crisis de autoridad institucional de las legislaciones neoliberales. Especialmente, esto ocurrió en los países del "primer mundo", donde la debacle económica perduró una década, creando grandes desigualdades y dificultades entre la ciudadanía. La consecuencia política más tangible fue el auge del malestar social, la proliferación de los discursos demagógicos y la emergencia de líderes con proyectos nacionalistas y autoritarios.

En el primer capítulo, "La mente copycat", se plantean algunas interrogantes sobre el descarrilamiento de los proyectos económico-legislativos surgidos del estado de ánimo colectivo de 1989. El pluralismo político occidental fue dejando paso a pensamientos reduccionistas y a grupos poblacionales polarizados ideológicamente. Aparentemente, las expectativas creadas fueron muchas con el horizonte de la opinión pública internacional de finales del siglo pasado, pero la degradación de los estándares de calidad de vida ha provocado un malestar creciente.

En el caso de la Unión Europea, después de absorber la mayoría de los países de Europa oriental, los planes de integración socioeconómica no se produjeron al ritmo esperado. La modélica Revolución de Terciopelo tuvo muchas consecuencias inesperadas, entre ellas, grandes bolsas de desigualdad social, que acabaron cristalizando en movimientos populistas (nacionalismo xenófobo y proteccionismo económico). Parece que los autores evitan términos como neofascismo y neonazismo; mientras que las contradicciones del neoliberalismo y los intensos debates intergeneracionales entre los teóricos de la economía reciben mucha atención en la parte final del capítulo. 
"La imitación como represalia" es el sugerente título del segundo capítulo, que da comienzo con una cita de La Rochefoucauld: "Las únicas buenas son aquellas que muestran la absurdez de los malos originales". Esta afirmación sintetiza el mensaje principal de suplantación de identidades e instrumentalización de discursos democráticos.

Estas páginas condensan el estilo más ensayístico de toda la publicación. La primera parte del capítulo está destinada a hablar del revisionismo ruso y de la propaganda del Kremlin, los autores conciben ambas maniobras como una estrategia para recuperar influencia sobre los antiguos Estados satélites. El discurso nacionalista de Putin es muy selectivo y plantea narrativas paralelas para explorar la posibilidad de implementar un "multilateralismo" exterior y la "multijustificación" interna. La creación de nuevos imaginarios democráticos es clave en todos estos apartados.

La estrategia de simular procedimientos democráticos, para legitimar y consolidar el poder institucional, constituye la base de cualquier planteamiento teórico a la hora de analizar la agenda de la era Putin. Los autores hacen un repaso pormenorizado y circunspecto de todos los elementos doctrinales implementados para consolidar el poder de Rusia en el inestable escenario internacional actual.

La disidencia interna y el liderazgo opositor, lejos de normalizarse, es criminalizado y perseguido sistemáticamente, aparentemente, a través de un proceso de judicialización vigilante y legislación disuasoria. Todo esto, sumado al liderazgo carismático de Putin, ha hecho despertar todas las alarmas entre los círculos oficialistas de Occidente.

En lo que respecta a la proyección exterior de Moscú, el revestimiento de acciones y valores democráticos responde a la búsqueda cíclica de consolidar un espacio de seguridad nacional. Del segundo capítulo se puede extraer la idea de que la Federación Rusa ha abandonado la idea soviética de derrotar y someter a Occidente, pero considera una necesidad el debilitamiento de los aliados en esta región del mundo (enfrentamientos entre países, disputas entre corporaciones e incremento de las fracturas sociales).

En el tercer capítulo, "La imitación como desposesión”, es un conjunto caleidoscópico de temas sobre la situación política durante la última década, tomando como punto de partida el proyecto de lo que se conoció en los noventa como "nuevo" orden mundial. En los dos primeros capítulos se habla sobre el proceso de languidecimiento y colapso del orden liberal internacional. 
El tercer capítulo se centra en las consecuencias del agotamiento del modelo y la búsqueda de una salida ordenada de esa situación. En el caso concreto de los Estados Unidos, Trump da un nuevo impulso al viejo lema de America first. La revolución MAGA ha supuesto un mayor grado de nacionalismo político, repliegue multilateral, proteccionismo económico, regeneración productiva y tradicionalismo cultural, en un momento en el que se consideraba a la deriva globalizadora como una humillación para el pueblo estadounidense.

El modelo de democracia del presidente Trump es, para muchos colectivos conservadores, la restauración de unos estándares de calidad perdidos tiempo atrás. La deriva del Partido Republicano ha supuesto, para muchos colectivos progresistas, una amenaza para el sistema democrático en sí mismo.

El presidente de los Estados Unidos siempre fue el faro de los líderes occidentales, pero Trump se ha convertido en un referente para los populismos que horadan los principios rectores del sistema democrático "liberal". Los autores consideran que la "Era de la Imitación Liberal" ha finalizado de la peor manera posible. También argumentan que la "Era de la Imitación Iliberal" solo acaba de comenzar. Parcialmente, Donald Trump y sus émulos son responsabilizados en este sentido.

En el apartado de la conclusión se hace una reflexión muy particular del liderazgo de las fuerzas occidentales durante la Guerra Fría y se analizan las alternativas del momento presente. En las últimas tres décadas, China ha construido un modelo económico de éxito y un sistema comercial expansivo y eficiente, se ha convertido en un ejemplo de gestión a imitar para algunos actores "tercermundistas".

En estas últimas páginas, se plantean preguntas retóricas formuladas para hacer reflexionar a la persona lectora, que en muchos casos suenan a advertencia, ante las posibles derivaciones ideológicas de la comunidad internacional. En dicha parte, que encaja a la perfección en el marco funcional de los ensayos críticos e interpretativos, los autores hacen un llamamiento a defender el sistema democrático liberal y el valor de la diversidad cultural. A la vez que no se establece ningún tipo de clasificación de las diferentes formas de concebir el liberalismo y tampoco se plantean grados de aplicación de este pensamiento. 


\section{Referencias}

Krastev, I. y Holmes, S. (2019). La luz que se apaga. Cómo Occidente ganó la Guerra Fría pero perdió la paz. Barcelona: Libros Debate. 342 páginas, ISBN: 978-84-17636-68-5 